\title{
miR-989 Is Required for Border Cell Migration in the Drosophila Ovary
}

\author{
Jan-Michael Kugler ${ }^{1}$, Ya-Wen Chen ${ }^{1}$, Ruifen Weng ${ }^{1,2}$, Stephen M. Cohen ${ }^{1,2 *}$ \\ 1 Institute of Molecular and Cell Biology, Singapore, Singapore, 2 Department of Biological Sciences, National University of Singapore, Singapore, Singapore
}

\begin{abstract}
microRNAs (miRNAs) are small non-coding RNAs that regulate gene expression by destabilizing target transcripts and/or inhibiting their translation. miRNAs are thought to have roles in buffering gene expression to confer robustness. miRNAs have been shown to play important roles during tissue development to control cell proliferation, differentiation and morphogenesis. Many miRNAs are expressed in the germ line of Drosophila, and functions have been reported for a few miRNAs in maintenance of stem cell proliferation during oogenesis. Here, we analyse the function of Drosophila miR-989 in oogenesis. miR-989 is abundant in ovaries. Mutants lacking miR-989 did not display gross abnormalities affecting egg chamber formation or maturation. However, the migration of the border cell cluster was severely delayed in miR-989 mutant egg chambers. We demonstrate that miR-989 function is required in the somatic cells in the egg chamber, not in germ line cells for border cell migration. Loss of miR-989 from a fraction of the border cell cluster was sufficient to impair cluster migration as a whole, suggesting a role in border cells. Gene ontology analysis reveals that many predicted miR-989 target mRNAs are implicated in regulating cell migration, cell projection morphogenesis, cell adhesion as well as receptor tyrosine kinase and ecdysone signalling, consistent with an important regulatory role for miR-989 in border cell migration.
\end{abstract}

Citation: Kugler J-M, Chen Y-W, Weng R, Cohen SM (2013) miR-989 Is Required for Border Cell Migration in the Drosophila Ovary. PLoS ONE 8(7): e67075. doi:10.1371/journal.pone.0067075

Editor: Burton B. Yang, University of Toronto, Canada

Received January 9, 2013; Accepted May 14, 2013; Published July 3, 2013

Copyright: (C) 2013 Kugler et al. This is an open-access article distributed under the terms of the Creative Commons Attribution License, which permits unrestricted use, distribution, and reproduction in any medium, provided the original author and source are credited.

Funding: Work in the SMC lab was supported by core funding from IMCB-Institute of Molecular and Cell Biology. Work in the JYY lab was supported by the Singapore National Research Foundation. The funders had no role in study design, data collection and analysis, decision to publish, or preparation of the manuscript.

Competing Interests: The authors have declared that no competing interests exist.

* E-mail: scohen@imcb.a-star.edu.sg

\section{Introduction}

miRNAs are small non-coding RNAs that function as regulators of gene expression in a wide range of biological contexts [1,2]. miRNAs associate with their target transcripts via partial complementary base pairing to target sites which are usually located in the target 3'UTR or in coding sequences [3,4]. In general, miRNAs act as negative regulators of gene expression at the post-transcriptional level by promoting target transcript destabilization and/or by reducing their translation $[1,2]$.

Border cells serve as a model system for the study of collective cell migration during Drosophila oogenesis [5,6,7]. Drosophila eggs mature in compound entities called egg chambers, which are comprised of 16 interconnected germ-line cells that are encapsulated by a monolayer of somatic follicle cells [8] (Fig. 1). One of the 16 germ-line cells differentiates as the oocyte, while the other 15 become polyploid nurse cells, which produce RNAs, proteins and organelles for incorporation into the oocyte to aid its maturation. The somatic follicle cells undergo a complex developmental and morphogenetic program that is tightly linked to germ line development and ultimately leads to the formation of the egg shell [7]. A subset of follicle cells, called border cells, has a special role during oogenesis, which involves an invasive, directed, cell migration. During stage 8 of oogenesis the border cells are specified at the anterior pole of the follicular epithelium and start to express the G/EBP transcription factor, Slow border cells (Slbo; Fig 1A). The border cells detach from the follicular epithelium and migrate as a cluster toward the oocyte during stage 9 to $10 \mathrm{~A}$
(Fig. 1B, C). At stage 10B, the border cell cluster has reached the anterior face of the oocyte and migrates laterally to its anterodorsal position (Fig. 1D). Specification of the border cells and the transition to coordinated cell migration involve several conserved signalling pathways and extensive remodelling of the cytoskeleton and cell adhesion properties $[5,6,7]$. The JAK/STAT pathway is required for border cell specification and for migration [9,10,11]. Ecdysone signalling regulates the timing of border cell specification $[12,13,14]$. Within the border cells, the receptor tyrosine kinases EGFR and PVR interpret guidance cues produced by the oocyte to direct anterior migration and later dorsal migration of the cluster $[15,16]$. Homophilic adhesive interactions between border cells and the nurse cells involving Cadherins are crucial for normal cluster migration [17].

In this report, we identify the miRNA miR-989 as a regulator of border cell migration. We show that border cell migration is delayed in miR-989 mutant egg chambers, and that this phenotype can be rescued by transgenic expression of the miRNA. Moreover, we demonstrate that miR-989 is active in the somatic cells of the egg chamber and required in border cells for efficient migration. Predicted targets encompass most of the pathways known to be involved in regulation of border cell migration.

\section{Results and Discussion}

Deep sequencing of an ovarian small RNA library identified miR-989 as the most abundant miRNA species in the Drosophila ovary, constituting $15.9 \%$ of all annotated sequencing reads [18]. 

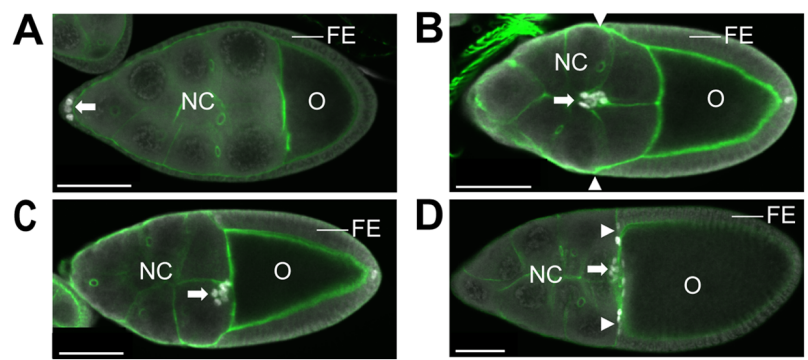

Figure 1. Morphology of mid-oogenesis egg chambers and border cell migration. Mid-oogenesis egg chambers labelled with Phalloidin (green) and border cell marker $\alpha$-Slbo (white). The germ line derived nurse cell (NC) cluster and oocyte (O) as well as the somatic follicular epithelium (FE), which encapsulates the germ line cells, are identified. A Stage S8 egg chamber. Slbo-positive border cells form in the FE anterior to the NC cluster (arrow). B Stage S9 egg chamber. The FE migrates towards the oocyte where it forms a columnar epithelium. Follicle cells stretch over the NC cluster to form a flat epithelium. The border cells (arrow) migrate through the NC cluster, roughly in parallel to the leading edge of the migrating external follicle cell sheet (arrowheads). C Stage S10A egg chamber. Migration of the border cell cluster and the migrating FE have essentially completed. D Stage S10B egg chamber. The centripetal follicle cells migrate over the anterior face of the oocyte (arrowheads).

doi:10.1371/journal.pone.0067075.g001
To test whether miR-989 has an important function during oogenesis, we generated a deletion allele (designated $m i R-989^{K O}$ ) by ends-out homologous recombination $[19,20]$. Deletion of the miR-989 gene was confirmed by PCR on genomic DNA (not shown). Ovaries derived from young females bearing the $m i R$ $989^{K O}$ allele in trans to a genomic deficiency $(D f(2 R)$ Exel7130) uncovering the miR-989 locus proved to be morphologically normal (not shown).

\section{Delayed border cell migration}

We observed that border cell migration was frequently delayed in $m i R-989^{K O} / D f(2 R)$ Exel7130 ovaries compared to controls and quantitated this phenotype during two stages of egg chamber development (Fig 2). During late stage S9 and S10A, we measured the distance between the leading border cell and the anterior-most cells in the sheet of follicle cells, as it migrates toward the oocyte (Fig. 2A). In all control genotypes, border cells kept pace with the advancing sheet of external follicle cells (Fig. 2B). In contrast, the border cell cluster lagged behind the follicular epithelium in homozygous miR-989 ${ }^{K O}$ egg chambers $(\mathrm{p}<0.001$ in comparison to the heterozygous control). Similar results were obtained when the $m i R-989^{K O}$ allele was placed in trans to two independent genomic deficiencies $(D f(2 R) 50 C-38$ and $D f(2 R)$ Exel7130) that uncover the miR-989 locus $(\mathrm{p}<0.001$ compared to all controls, Fig $2 \mathrm{~B})$. In wild type egg chambers, border cells typically have reached the oocyte by stage $10 \mathrm{~B}$ and have begun to migrate toward their final
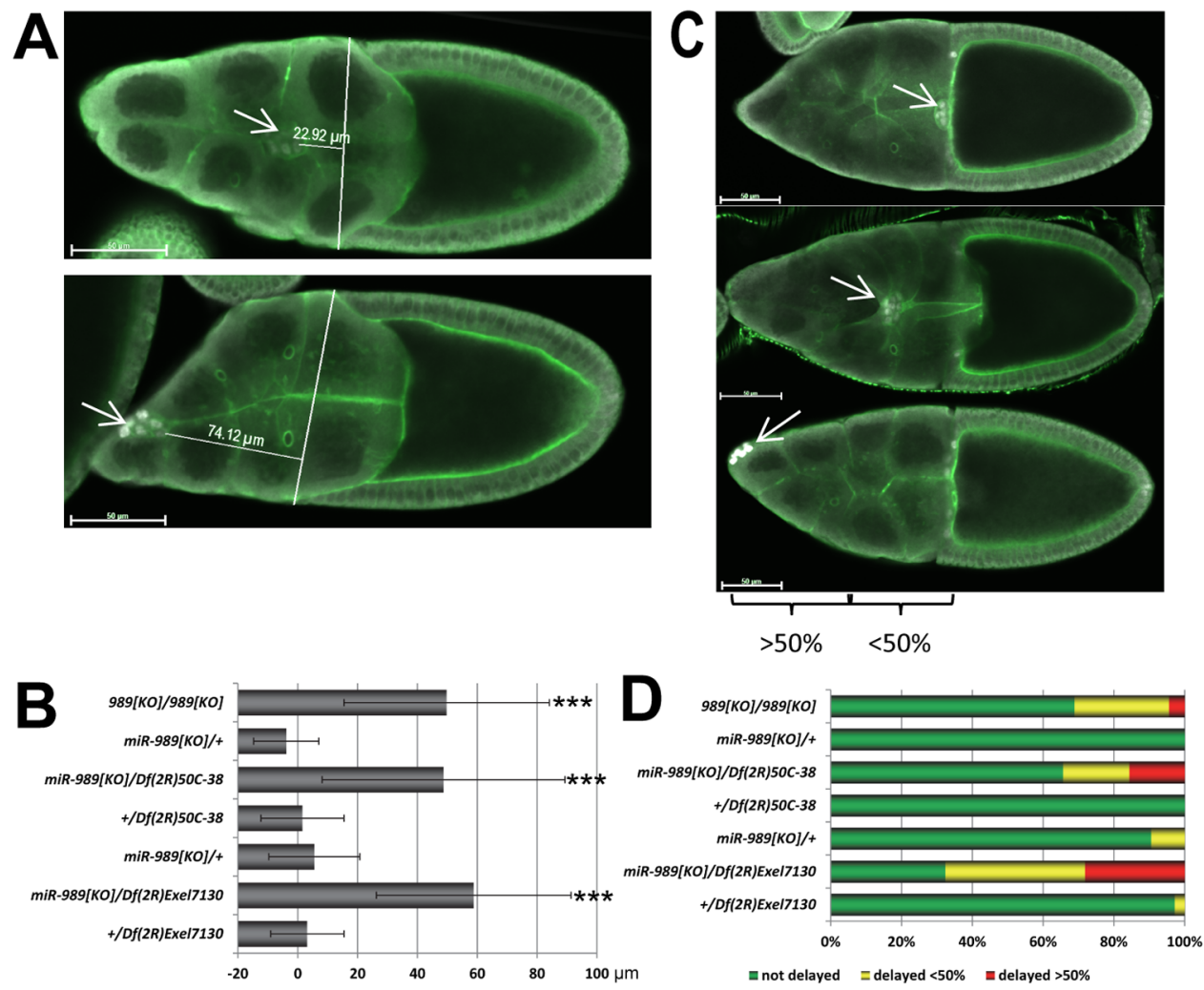

Figure 2. Border cell migration is delayed in miR-989 mutant egg chambers. A Late stage 9 egg chambers, labelled with $\alpha$-Slbo (white) and Phalloidin (green). Border cell clusters are highlighted by arrows. In miR-989 mutant egg chambers, border cells were frequently delayed relative to the migrating main body follicular epithelium. B Quantification of the border cell migration phenotype in stage 9 and stage $10 \mathrm{~A}$ egg chambers. Border cell migration is delayed in miR-989 mutant egg chambers compared to heterozygous control egg chambers $(* * * p<0.001)$. X-Axis labels are in $\mu \mathrm{m}$, error bars denote standard deviation. C Stage 10B egg chambers, labelled with $\alpha$-Slbo (white) and Phalloidin (green). Border cell clusters are highlighted by arrows. Frequently, border cells had not reached the oocyte by this stage in miR-989 mutant egg chambers. D Quantification of the border cell migration phenotype in stage 10B egg chambers.

doi:10.1371/journal.pone.0067075.g002 

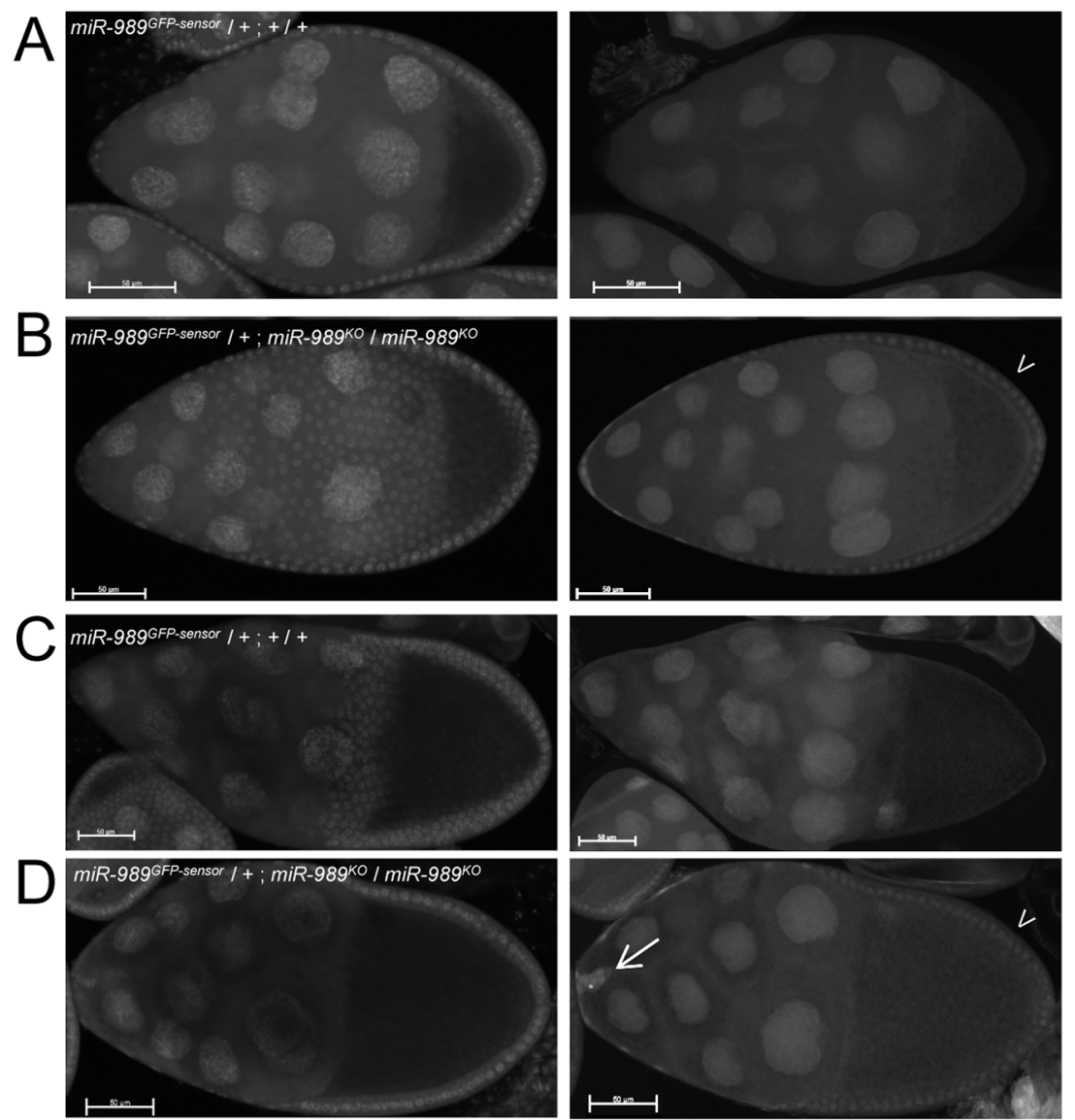

DAPI

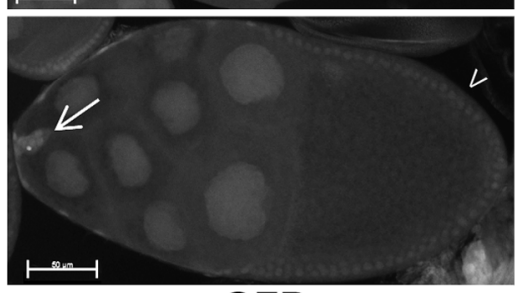

GFP

Figure 3. A miRNA sensor suggest somatic miR-989 activity. Stage 8 (A, B) and Stage 10A (C, D) eggchambers expressing a miR-989 GFP sensor in a wild type (A, C) or in a miR-989 mutant background (B, D). While the GFP sensor was expressed at similar levels in the germ line cells in both genetic backgrounds, it was not detectable in the somatic follicle cells in the wild-type egg chambers. However, follicle cells were positively labelled by the sensor in the absence of miR-989. Note that the sensor also labels the border cells in (D) (arrow).

doi:10.1371/journal.pone.0067075.g003

anterodorsal position. In S10B egg chambers lacking miR-989, border cells were frequently found within the nurse cell compartment. We quantitated this phenotype by scoring whether the border cells had reached the oocyte ("not delayed"), or whether they were still found in the anterior half (" $>50 \%$ delayed") or posterior half (" $<50 \%$ delayed") of the nurse cell compartment (Fig. 2C). Using this scoring scheme, we found that most border cell clusters in the heterozygous control egg chambers had arrived at the oocyte at stage 10B. Over $1 / 3$ of border cell clusters derived from miR-989 mutant females were delayed. These observations suggested that miR-989 is required for some aspect of border cell migration towards the oocyte.

\section{miR-989 activity in somatic cells}

Border cell migration depends on guidance signals from germline cells, signal interpretation by the border cells to produce directed migration and interaction between the two cell types to allow movement of the border cell cluster on and between the nurse cells $[5,6,7]$. We therefore asked whether miR-989 was acting in the somatic cells or germ line cells of the ovary. As a first step to address this question, we generated transgenic flies that express a miRNA sensor [21] for miR-989. This sensor encodes eGFP expressed ubiquitously under control of a tubulin promoter, followed by a 3' UTR containing two target sites for miR-989. In a wild type background, sensor-directed GFP was present in all the germ line cells, but GFP was not detected in the somatic follicle cells (Fig. 3 A, G). In the miR-989 mutant background, a homogenous GFP signal was also observed in all somatic cells, including the border cell cluster while GFP expression in the germ line cells was unchanged (Fig. 3B, D, arrows). This suggests that miR-989 is predominantly active in the somatic follicle cells.

\section{miR-989 is required in somatic cells for efficient border cell migration}

The predominant pattern of follicle cell expression suggested that miR-989 activity may be required in the follicle cells or the border cells to promote normal border cell migration. To test this idea, we generated mosaic egg chambers that were partially wildtype and partially mutant for $m i R-989$ and scored the migratory behaviour of the border cells. We found that wild-type border cells migrated normally when the germ line cells lacked miR-989 (Fig. 4A, left panel). In contrast, migration was delayed when somatic cells including the border cell cluster were mutant for $m i R$ 989 but the germ line was wild-type (Fig. 4B, right panel). The quantification of these observations is shown in Figure $4(\mathrm{~B}, \mathrm{C})$. Border cell migration was delayed in a statistically significant manner $(\mathrm{p}<0.001)$ when all cells in the border cell cluster were mutant for miR-989, but the germ cells were wild-type. We did not 

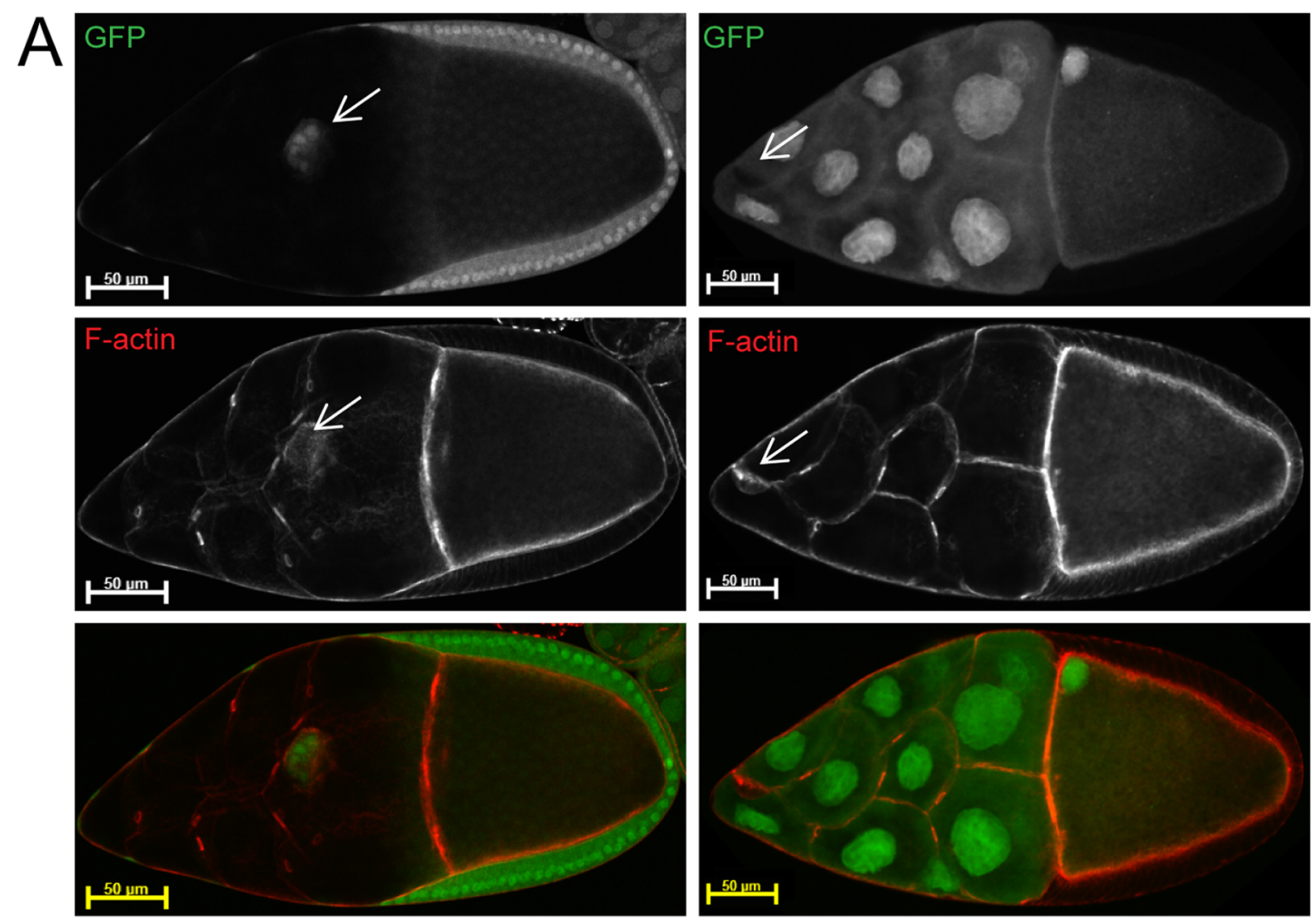

Border cells: wild type

Germ line: $\operatorname{miR}-989^{K O}$

Border cells: miR-989ko

Germ line: wild type
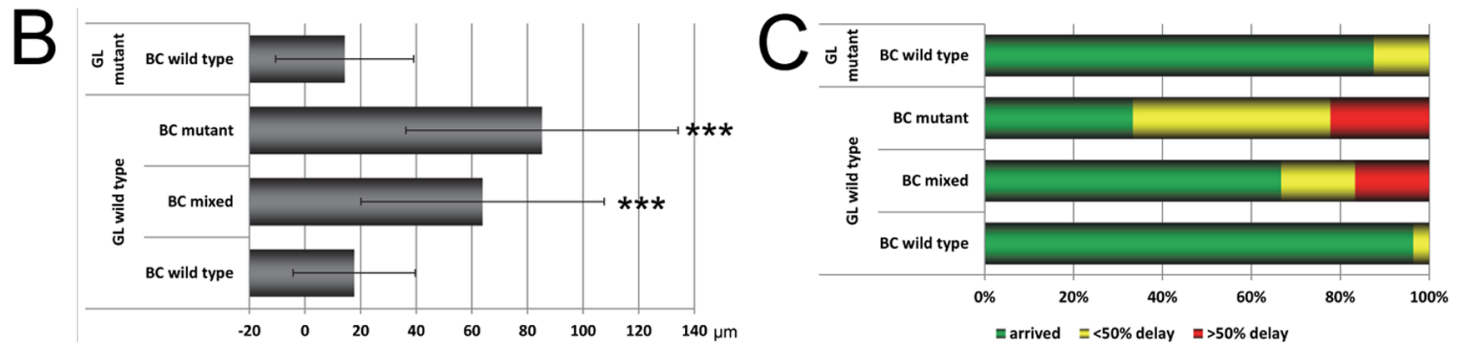

Figure 4. Clonal analysis demonstrates a somatic requirement for miR-989 for normal border cell migration. A Genetic mosaics: wild type cells are labelled by nuclear GFP (upper panel) while miR-989 mutant cells do not express GFP. The middle panels are labelled with phalloidin to highlight the F-actin cytoskeleton. The two egg chambers shown represent extreme cases of mosaicism. In the stage $10 \mathrm{~A}$ egg chamber on the left all somatic cells are wild-type (GFP positive), while all germ line cells are mutant for miR-989 (GFP negative). The border cells were not delayed in their migration. In the stage 10A egg chamber shown on the right all germ line cells are wild type (GFP positive) while all somatic cells are mutant for miR989 (GFP negative). Border cell migration was strongly delayed. B Quantification of the migration defects in migrating border cells in late stage 9 and stage 10A egg chambers. GL denotes germ line, and BC border cells. Migration was strongly delayed if the border cells were mutant, but not if the germ line cells lacked miR-989. We observed a population of border cell clusters that were partially wild-type and partially mutant for miR-989. In these cases, border cell migration was also delayed in comparison to controls. ${ }^{* * *}$ indicates $p<0.001$. C Quantification of the migration defects in stage 10B egg chambers. Wild type border cells migrated normally when the germ line was mutant for miR-989. Conversely, miR-989 mutant border cells were delayed when the germ line was wild type.

doi:10.1371/journal.pone.0067075.g004

observe a statistically significant delay in egg chambers in which the border cells were wild-type but the germline was mutant for $m i R-989$. These results provide evidence that $m i R-989$ is required in somatic cells for normal migration, but dispensable in the germline.

We also observed egg chambers in which the border cell cluster was partially wild type and partially mutant for miR-989 while germ line was wild-type (Fig. 4B, C). Intriguingly, such mixed genotype border cell clusters were also delayed in their migration $(\mathrm{p}<0.001$, compared to control egg chambers). The delay was similar in magnitude to that of completely mutant clusters $(\mathrm{p}>0.1$ between completely and partially mutant border cell clusters). This suggests that lack of miR-989 in just some border cells is sufficient to cause delays affecting the entire cluster. In other words, the presence of wild-type border cells cannot compensate for the lack of miR-989 in some cells. However, we do not exclude the possibility that loss of miR-989 from other somatic cells might contribute to the border cell migration phenotype. 

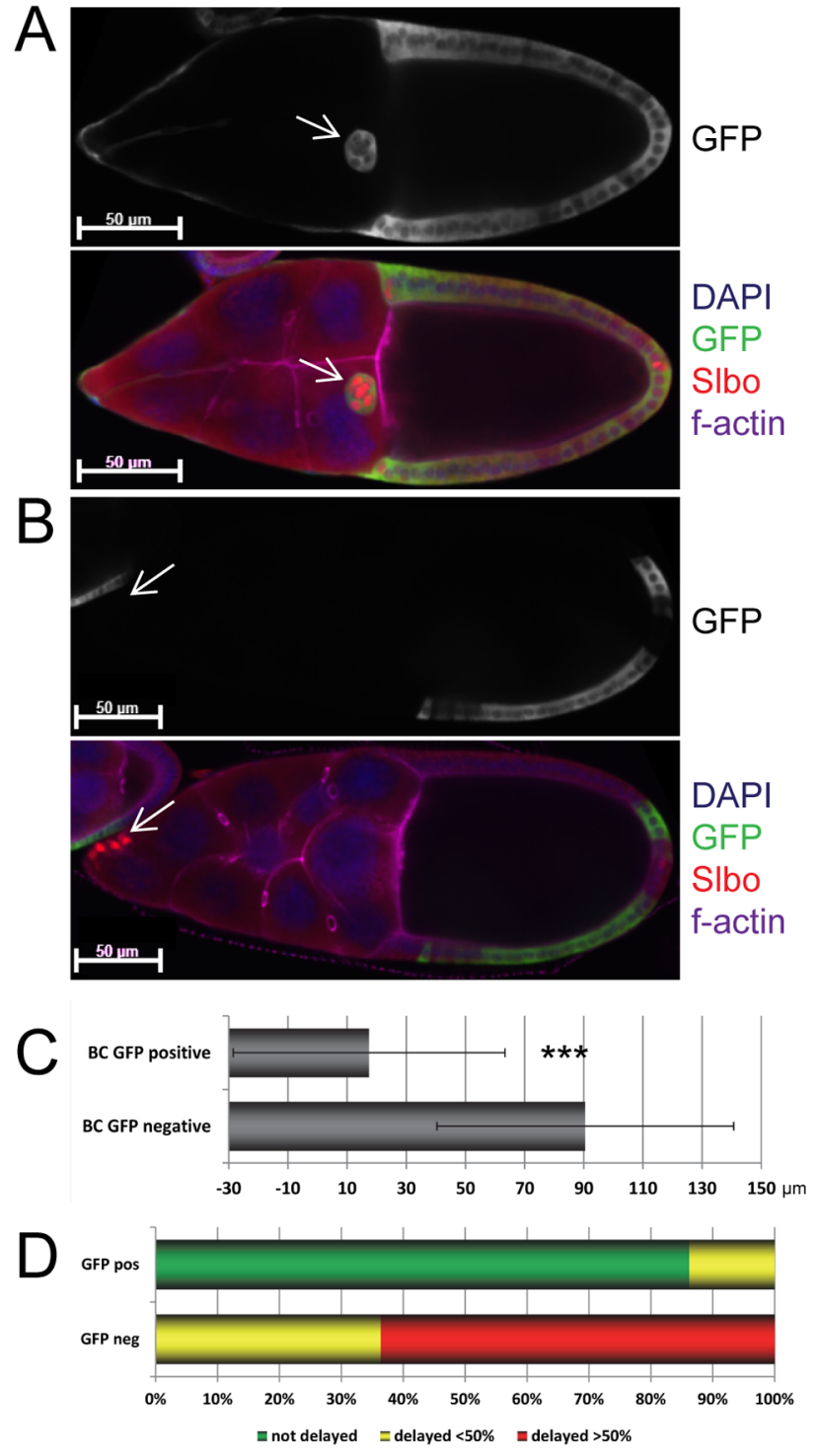

Figure 5. The border cell migration phenotype was rescued by transgenic expression of miR-989. A-D Expression of miR-989 in border cells in a miR-989 mutant background suppressed the delayed border cell migration phenotype. Presence of GFP indicates transgenic miR-989 expression. A Example of a mosaic egg chamber in which miR989 and GFP were expressed in border cells and border cell migration was normal. B Example of a mosaic egg chamber in which miR-989 and GFP were not expressed in border cells, and border cell migration was drastically delayed. C Quantification of border cell migration progress in mosaic stage 9 and stage $10 \mathrm{~A}$ egg chambers. Mosaic egg chambers in which border cells were mutant for miR-989 were strongly delayed in their migration. Transgenic expression of miR-989 in border cells of sibling mosaics partially suppressed the delayed border cell phenotype. D Quantification of border cell migration progression in mosaic stage $10 \mathrm{~B}$ egg chambers. Border cells transgenically expressing miR-989 in a miR-989 mutant background most often reached the oocyte by this stage. In contrast, miR-989 and GFP negative border cells in sibling mosaics were strongly delayed.

doi:10.1371/journal.pone.0067075.g005

\section{The border cell migration defect can be rescued by transgenic miR-989 expression}

To confirm that lack of $m i R-989$ was responsible for the border cell migration defects described above, we expressed miR-989 from an UAS transgene. Expression with the Slbo-Gal4 driver did not rescue border cell migration in a $m i R-989^{K O}$ mutant background. In contrast, miR-989 expression under the control of a heat-shock inducible actin-flip-out-Gal4 cassette was able to rescue. This technique allowed us to restore miRNA expression in subsets of cells that were positively marked by presence of GFP. GFPnegative border cell clusters lacking miR-989 were delayed in their migration (Fig. 5A, C, D), whereas GFP-positive border cell clusters with transgenic miR-989 expression migrated almost normally (Fig. 5B, C, D; p $<0.001$ ). This demonstrates that loss of $m i R-989$ was responsible for the delayed border cell migration phenotype in the miR-989 mutant egg chambers.

miR-989 is expressed in the somatic cells throughout egg chamber development. In the flip-out clonal experiment, Gal4 expression was induced well before the onset of border cell migration. The Slbo gene turns on in stage 8, shortly before border cell migration begins. Expression of a miRNA under Slbo-Gal4 control is expected to take some time to effectively repress its targets. Comparing the Slbo-Gal4 and flip-out clonal rescue results suggests that miR-989 may be required from the onset of border cell migration.

\section{predicted miR-989 targets}

miR-989 target predictions were obtained from TargetScanFly [22], TargetScanFly ORF [3], MinoTar [3] and miRNA.org [23]. Together, these algorithms identify 724 non-redundant candidate target transcripts. We performed process GO term analysis using the GOrilla interface [24] to identify targets that might be involved in regulating border cell migration (Figure $\mathrm{S} 1$ and Table $\mathrm{S} 1$ ). This analysis revealed 163 enriched GO terms. Among these were potentially informative GO term nodes with lower GO term hierarchy level which were significantly enriched: 'cell migration' (enriched 2.8 fold, $\mathrm{p}=1.6 \times 10^{-7}, 31$ putative target genes), 'cell adhesion' (enriched 2.6 fold, $\mathrm{p}=7.4 \times 10^{-7}, 25$ putative targets), 'cell projection morphogenesis' (enriched 2.4 fold, $\mathrm{p}=8.6 \times 10-5$, 23 putative targets), 'transmembrane receptor protein tyrosine kinase signaling pathway' (enriched 2.7 fold, $p=2.9 \times 10^{-4}, 16$ putative targets) and 'response to ecdysone' (enriched 4.6 fold, $\mathrm{p}=6.3 \times 10^{-4}, 7$ putative targets). miRNAs typically downregulate their targets, in part through target transcript destabilization. It is therefore expected that target levels would increase in miRNA mutant cells. The ovary has two main cell types: somatic and germ line. Somatic cells, where miR-989 is expressed, comprise a small fraction of the total tissue. Because the border cells are only a small fraction of the somatic cells, we have not attempted to validate upregulation of candidate targets in the miR-989 mutant border cells by monitoring target RNA levels.

Predicted miR-989 targets associated with the GO terms 'cell migration' and 'regulation of cell projections' are obvious candidates whose de-regulation may cause border cell migration phenotypes. The receptor tyrosine kinase receptors EGFR and Pvr are required in the border cells to receive guidance queues that direct cluster migration towards the oocyte $[15,16]$. Upregulation of negative regulators of these signalling pathways could delay border cell migration. Ecdysone signaling play a crucial role in regulating the timing of border cell specification, and defects in border cell specification can delay migration [12,13,14].

Predicted miR-989 targets associated with the GO term 'cell adhesion' may be particularly interesting. Border cells migrate collectively in a coordinated fashion, and migration events critically depend on remodelling events that change the adhesive properties of the migrating cells $[5,6,7]$. In the case of the border cell cluster, border cells have to give up their connection to neighbouring follicular epithelial cells when they initiate migration 
towards the oocyte. Likewise, while they do need to adhere to the nurse cell membranes, through homophilic interactions of DEcadherin [17] they must not adhere too tightly in order to migrate efficiently. In this light, it is interesting to note that loss of miR-989 from a subset of border cells is sufficient to cause border cell migration delays. Since border cells adhere tightly to each other $[5,6,7]$, it is easily conceivable that few cells that adhere too tightly to the follicular epithelium or the migration substrate would impair migration of the entire cluster. This has been documented for clusters partially mutant for Slbo [25], which affects border cell specification and for clusters partially mutant for DE Cadherin [17]. miR-989 could act on multiple targets including those affecting the processes discussed above to permit normal border cell migration.

\section{Materials and Methods}

\section{Fly stocks}

The $m i R-989^{K O}$ allele was generated using pRMCE as described $[19,20]$. The UAS-miR-989 transgene was cloned into pUAST.attB-SLIC [26] and integrated into the landing site $86 \mathrm{Fb}$ to generate pT-989@Fb. To generate the miR-989 sensor, oligos encoding two perfect miR-989 binding sites were annealed and cloned into the tub>eGFP transgene [21]. The sensor was then transformed by P-element mediated germ line transformation and individual transgenic lines were established by standard procedures. Oligonucleotide sequences are available on request. The genomic deficiencies $D f(2 R)$ Exel7130 and $D f(2 R) 50 C-38$ and the hsFLP; FRT42A ubiGFPnls strain were obtained from the Bloomington stock centre. Actin-Flipout-Gal4 (AFG) UAS-10xGFP was obtained from the Rørth lab [27]. For mosaic analysis (Fig. 4), males bearing a recombinant FRT $42 A$ miR- $989^{K O}$ chromosome were crossed to hsFLP; FRT42A ubiGFPnls virgins and offspring third instar larvae were heat-shocked for $1-2 \mathrm{~h}$ at $37^{\circ} \mathrm{C}$. For the rescue analysis, third instar larvae of the genotype $h s F P /+$; $m i R$ 989KO/miR-989 ${ }^{K O}$; pT-989@Fb/AFG,10xGFP were heat-shocked at $37^{\circ} \mathrm{C}$ for $1-2 \mathrm{~h}$ and ovaries were then dissected from $2-3 \mathrm{~d}$ old adult females for analysis.

\section{Immunostainings and Microscopy}

1-3d old mated females were collected and placed on wet yeast for $12-24 \mathrm{~h}$ prior to dissection. Ovaries were dissected into Schneider's S2 cell medium supplemented with $50 \mu \mathrm{l} / \mathrm{ml}$ FCS and lul $10 \mathrm{mg} / \mathrm{ml}$ Insulin (Sigma) per ml medium. Following dissection in $4 \%$ formaldehyde, samples were rinsed and washed in PBST (PBS $+0.3 \%$ Triton-X), then blocked in PBSTB $(\mathrm{PBST}+3 \% \mathrm{BSA})$

\section{References}

1. Bartel DP (2009) MicroRNAs: target recognition and regulatory functions. Cell 136: 215-233.

2. Bushati N, Cohen SM (2007) microRNA functions. Annu Rev Cell Dev Biol 23: 175-205.

3. Schnall-Levin M, Zhao Y, Perrimon N, Berger B (2010) Conserved microRNA targeting in Drosophila is as widespread in coding regions as in 3'UTRs. Proc Natl Acad Sci U S A 107: 15751-15756.

4. Stark A, Brennecke J, Russell RB, Cohen SM (2003) Identification of Drosophila MicroRNA targets. PLoS Biol 1: E60.

5. Montell DJ (2003) Border-cell migration: the race is on. Nat Rev Mol Cell Biol 4: 13-24.

6. Rorth P (2009) Collective cell migration. Annu Rev Cell Dev Biol 25: 407-429.

7. Wu X, Tanwar PS, Raftery LA (2008) Drosophila follicle cells: morphogenesis in an eggshell. Semin Cell Dev Biol 19: 271-282.

8. Bastock R, St Johnston D (2008) Drosophila oogenesis. Curr Biol 18: R10821087.

9. Beccari S, Teixeira L, Rorth P (2002) The JAK/STAT pathway is required for border cell migration during Drosophila oogenesis. Mech Dev 111: 115-123. and incubated overnight in primary antibody (rat anti slbo 1:500) at $4{ }^{\circ} \mathrm{C}$. The ovaries were then extensively washed with PBSTB at room temperature and incubated overnight in secondary antibodies (AlexaFluor antibodies (Invitrogen), 1:250 of the 1:1 glycerol stocks). If applicable, Alexa633- or Alexa555-conjugated Phalloidin was added at 1:150 (Invitrogen). Samples were then washed extensively and counterstained with DAPI. For sensor analysis, ovaries were fixed as above, extensively washed in PBST and counterstained with DAPI. Images were obtained either on a Zeiss Axio Imager 2.0 or a Zeiss LSM700 confocal microscope. Images were analysed with Zeiss Axiovision software. Graphs and statistical analysis were done with Microsoft Excel.

\section{GO term analysis of predicted miR-989 targets}

miR-989 target lists were obtained from TargetScanFly [22], TargetScanFly ORF [3], MinoTar [3] and miRNA.org [23], standardized to CG gene name annotation using Flybase [28], pooled and then pruned of redundant entries using Excel. A genome-wide list of CG annotated genes in Drosophila melanogaster was obtained from Flybase as a reference pool. GO term analysis was performed using the GOrilla platform (http://cbl-gorilla.cs. technion.ac.il/), querying all Drosophila ontologies using the $m i R$ 989 target predictions as target set and all Drosophila genes as a background set.

\section{Supporting Information}

Figure S1 GO term analysis of predicted miR-989 targets. Figure $\mathrm{S} 1$ shows a directed acyclic graph created by the GOrilla interface. It shows GO term enrichment of predicted miR-989 targets (color coded).

(JPG)

Table S1 GO terms enriched among the predicted $m i R$ 989 targets. Table $\mathrm{S} 1$ shows an annotated list of the GO terms that are enriched among predicted miR-989 targets.

(XLSX)

\section{Acknowledgments}

We thank Pernille Rørth and HsinHo Sung for insightful discussion and advice, as well as for Slbo antibodies and fly strains.

\section{Author Contributions}

Conceived and designed the experiments: JMK SMC. Performed the experiments: JMK. Analyzed the data: JMK SMC. Contributed reagents/ materials/analysis tools: YC RW. Wrote the paper: JMK SMC.
10. Silver DL, Geisbrecht ER, Montell DJ (2005) Requirement for JAK/STAT signaling throughout border cell migration in Drosophila. Development 132: 3483-3492.

11. Silver DL, Montell DJ (2001) Paracrine signaling through the JAK/STAT pathway activates invasive behavior of ovarian epithelial cells in Drosophila. Cell 107: 831-841.

12. Bai J, Uehara Y, Montell DJ (2000) Regulation of invasive cell behavior by taiman, a Drosophila protein related to AIB1, a steroid receptor coactivator amplified in breast cancer. Cell 103: 1047-1058.

13. Godt D, Tepass U (2009) Breaking a temporal barrier: signalling crosstalk regulates the initiation of border cell migration. Nat Cell Biol 11: 536-538.

14. Jang AC, Chang YC, Bai J, Montell D (2009) Border-cell migration requires integration of spatial and temporal signals by the BTB protein Abrupt. Nat Cell Biol 11: 569-579.

15. Duchek P, Rorth P (2001) Guidance of cell migration by EGF receptor signaling during Drosophila oogenesis. Science 291: 131-133.

16. Duchek P, Somogyi K, Jekely G, Beccari S, Rorth P (2001) Guidance of cell migration by the Drosophila PDGF/VEGF receptor. Cell 107: 17-26.

17. Niewiadomska P, Godt D, Tepass U (1999) DE-Cadherin is required for intercellular motility during Drosophila oogenesis. J Cell Biol 144: 533-547. 
18. Czech B, Malone CD, Zhou R, Stark A, Schlingeheyde C, et al. (2008) An endogenous small interfering RNA pathway in Drosophila. Nature 453: 798802.

19. Chen YW, Weng R, Cohen SM (2011) Protocols for use of homologous recombination gene targeting to produce microRNA mutants in Drosophila. Methods Mol Biol 732: 99-120.

20. Weng R, Chen YW, Bushati N, Cliffe A, Cohen SM (2009) Recombinasemediated cassette exchange provides a versatile platform for gene targeting: knockout of miR-31b. Genetics 183: 399-402.

21. Brennecke J, Hipfner DR, Stark A, Russell RB, Cohen SM (2003) bantam encodes a developmentally regulated microRNA that controls cell proliferation and regulates the proapoptotic gene hid in Drosophila. Cell 113: 25-36.

22. Ruby JG, Stark A, Johnston WK, Kellis M, Bartel DP, et al. (2007) Evolution, biogenesis, expression, and target predictions of a substantially expanded set of Drosophila microRNAs. Genome Res 17: 1850-1864.
23. Betel D, Wilson M, Gabow A, Marks DS, Sander C (2008) The microRNA.org resource: targets and expression. Nucleic Acids Res 36: D149-153.

24. Eden E, Navon R, Steinfeld I, Lipson D, Yakhini Z (2009) GOrilla: a tool for discovery and visualization of enriched GO terms in ranked gene lists. BMC Bioinformatics 10: 48.

25. Rorth P, Szabo K, Texido G (2000) The level of C/EBP protein is critical for cell migration during Drosophila oogenesis and is tightly controlled by regulated degradation. Mol Cell 6: 23-30.

26. Szuplewski S, Kugler JM, Lim SF, Verma P, Chen YW, et al. (2012) MicroRNA transgene overexpression complements deficiency-based modifier screens in Drosophila. Genetics 190: 617-626.

27. Poukkula M, Cliffe A, Changede R, Rorth P (2011) Gell behaviors regulated by guidance cues in collective migration of border cells. J Cell Biol 192: 513-524.

28. McQuilton P, St Pierre SE, Thurmond J (2012) FlyBase 101-the basics of navigating FlyBase. Nucleic Acids Res 40: D706-714. 